\title{
Current concepts in the management of the axilla in early breast cancer: proposed standard of practice in Sri Lanka
}

\author{
Naomal M A Perera \\ Lanka Hospitals, Colombo, Sri Lanka
}

Keywords: Sentinel lymph node dissection; sentinel lymph node; early breast cancer; axilla

\begin{abstract}
Despite breast conservation surgery frequently been done for early breast cancers, mastectomy is still considered as the gold standard in surgical management. However, the management of the axilla has been published following many randomized clinical trials and debated at numerous forums and has gradually become less extensive than the radical axillary clearance done in the past. The reasons are multifactorial and could be attributed for the technological advances in surgery, radiology, systemic therapy and specially radiation therapy. Hence, intense attentiveness on the preoperative status of the axilla has become important in the management of early breast cancer at present.
\end{abstract}

The global recommendation in managing the axilla for early breast cancer is sentinel lymph node dissection. Currently, with the availability of mammography in most provinces in Sri Lanka, more early cancers with clinically node negative axilla are been detected. Unfortunately, the sentinel lymph node dissection been offered for them are limited, mainly due to the absence of insight on the technique. The current recommendations, and techniques to overcome these recommendations with the limited resources in our country are reviewed in this article.

\section{Introduction}

The pathological status of the axilla is considered as one of the major prognostic factors in the management of Breast Cancer. It has been the gold standard to offer level 1 and 2 clearance of the axillary nodes for all stages of breast cancer in the past. Although been documented that over $80 \%$ of women who undergo standard axillary lymph node dissection (ALND), have at least one post-operative complication of the arm and psychological distress [1], is still been routinely practiced in many centres in Sri Lanka. As an alternative for ALND, blue

Correspondence: Naomal Perera

E-mail: dr.naomal@gmail.com

Received: 27-11-2019 Accepted: 29-12-2019

(iD) https://orcid.org/0000-0002-4101-9010 DOI: http://doi.org/10.4038/sljs.v37i4.8650 dye sentinel node mapping in breast cancer was first published by Guiliano et al in 1994 [2]. Since then numerous randomized clinical trials and multicentre studies have concluded that the Sentinel Lymph Node Dissection (SLND) has supplanted the ALND as the standard approach to the axilla in patients with clinically node negative breast cancer [3-5]. While justifying the use of SLND as the new standard in the assessment of the axilla in clinically node negative patients, use of this method has shown to reduce the postoperative morbidity and long-term complications which were associated with ALND [6].

\section{Indication and contraindications of SLND}

Sentinel lymph node (SLN) mapping is not for all patients with Breast Cancer. Early Breast Cancer with clinically node negative axilla is the most important criteria that needs fulfilling to offer SLND. Hence it is an utmost importance and mandatory to assess the axillary status clinically as well as by ultrasound image guided biopsy at triple assessment to select eligible patients for SLND. However, in a systematic review and meta-analysis done to assess the value of preoperative ultrasound-guided axillary lymph node biopsy, only about $50 \%$ of women with axillary involvement was identified preoperatively [7]. And one in four women with an ultrasound-guided biopsy "proven" negative axilla were later found positive at SLND [7].

Clinically positive axilla and inflammatory cancer (T4b) are considered as absolute contraindications for SLND, and up to level 2 ALND should be offered in all such instances. However, neoadjuvant chemotherapy (NACT) given for advanced primary of the breast needs be studied individually and the decision on SLND or an ALND should be taken by the multi-disciplinary team. If the axilla was found clinically negative prior to and remains negative following NACT, SLND can be offered, though the sentinel lymph node identification rate (IR) can be low as 89.6 percent with a false negative rate (FNR) high as 14.2 percent [8]. Although the long-term consequences of high FNR have not been studied, the surgeons are advised to perform a meticulous SLND and to obtain more sentinel nodes to minimize the error rate for patients who had NACT. 
Regardless of clinically negative axillae prior to NACT, and if found to have progression of axillary status to be positive while on treatment, warrants a proper level 2 ALND [9].

For patients with clinically involved nodes with or without image guided positive biopsy and remains to be the same following NACT needs proper ALND [9]. But for patients who get converted to clinically node negative stage following NACT, an ALND can be avoided. However, these patients who undergo SLND require more than two sentinel lymph nodes to be sampled to confirm as a negative axilla due to high FNR associated with NACT.

Multicentric disease is not a contraindication for SLND as studies of breast lymphatic drainage indicate that all quadrants of the breast drain into the same lymph node basin [10]. Thus, subareolar and intradermal injection, rather than peritumoural injection of the radio isotope colloid and the blue dye is practical for patients with multicentric disease.

Previous breast surgery for benign diseases or a previous axillary surgery could disrupt or alternate the normal lymphatic drainage of the breast results in increase false negative rates. Although two previous studies have found a feasibility and accuracy of SLND following previous excisional breast biopsies [11,12], the author has noticed it otherwise. However, there are reports of successful second SLND for local recurrences in the breast and who had breast conservation surgery in the past [13]. Hence due to contradictory arguments on SLND after axillary surgery with possible disruption of the lymphatics a lymphoscintigraphy can be performed to identify the lymphatic drainage prior to SLND.

Lastly, a technically failed SLN detection at any stage of the disease needs a full ALND to fulfil proper axillary treatment.

\section{Validation and technique of SLND}

SLND has been developed and validated over a period of three decades. The National Comprehensive Cancer Network (NCCN) guidelines (version 3.2019) essentially recommends an experienced SLN team for the use of mapping and surgical excision. Although there are controversial publications regarding the number of cases required to ensure safety and reasonable low failure and accuracy rates, surgical training requirements and standardization of evidence-based techniques are driving the procedural accuracy to be over $97 \%$ and FNR less than $5 \%$ [14].

Despite variability in selection criteria and technique, a sentinel lymph node is consistently identified in approximately 96 percent of patients with FNR of 7.3 percent in most studies including the systematic review of 69 trials of SLNB, including 8059 patients. Nevertheless, American
Society of Clinical Oncology recommends less than $5 \%$ as the acceptable FNR in SLND [15].

Each surgeon needs to identify the best technique to practice following validation of his or her method with the resources available within their institution, which should be adopted from an accepted guideline.

\section{SLND in Sri Lanka}

Presently SLND is been practiced in many Teaching / Tertiary hospitals in Sri Lanka though some may not be following the accepted international guidelines. Further there is no proper consensus or an accepted protocol in Sri Lanka other than to follow NCCN guidelines. It is important to realize that the consequences of an invalidated technique or not following accepted guidelines could under stage the axilla and undertreat the patient resulting in poor outcomes.

SLND can be performed with the blue dye, radioactive colloid or with both tracers. Using a combination of blue dye and radioactive colloid resulted in a significantly higher success rate and lower FNR in sentinel node mapping compared with using blue dye alone [16]. In Sri Lanka many centres use only methylene blue to localize the SLN due to lack of resources. The meta-analysis of 18 studies with 1559 patients done by Jiyu $\mathrm{Li}$ et al, have reported 91 percent identification rate by methylene blue alone. Hence the authors have concluded by commenting that use of methylene blue alone to localize a sentinel node did not differ substantially when compared with combination method or use of other blue dyes [17]. Unfortunately, the same meta-analysis reported a higher FNR of 13 percent which was far above the accepted false negative rate recommended by the American Society of Clinical Oncologist [15].

Therefore, when methylene blue dye alone is used for SLN mapping, it is recommended attempting to identify more sentinel nodes, removing any hard or large nodes found adjacent to them, only to perform routinely after the technique been validated by the surgeon and selecting patients with smaller tumors.

Comparatively methylene blue has a lower molecular weight $(319.85 \mathrm{~g} / \mathrm{mol})$ than other commonly used blue dyes (Isosulfan blue $566.7 \mathrm{~g} / \mathrm{mol}$, patent blue $582.7 \mathrm{~g} / \mathrm{mol}$ ). Further methylene blue does not have sulfonic acid group in its structure which can combine with amino groups on the protein surface to make a macro particle that get trapped easily within a lymph node [18]. Hence there is always a possibility that methylene blue can escape through the sentinel node and get trapped within the 2 nd or the 3 rd node in line. This explains why methylene blue SLN mapping gives a higher false negative rate. But use of either $2 \mathrm{ml}$ or $5 \mathrm{ml}$ of methylene blue alone or the optimal injection site has not shown any 
significant difference in the identification rate or the FNR in the meta-analysis done by Jiyu Li.

\section{Isotopes}

Medical isotopes are limited only to very few tertiary hospitals in Sri Lanka with constrained supplies of technetium sulfur colloid to localize the sentinel node routinely. Although it is time and money consuming, it overweighs by the advantage of the gamma probe signalling the 'hot' node identifying even through the fat in the axilla. Therefore, it is advisable to use isotopes with the blue dye if facilities are freely available to exploit the advantages of the technology.

\section{Sentinel nodes}

Successful sentinel lymph node identification by blue dye is defined as the identification of any 'blue node' or any 'nonblue node' with a blue afferent lymphatic [19]. Hence gentle palpation through the axillary incision should be done once the blue stained lymphatic channels are exposed. It is recommended to remove more than 2 sentinel nodes to minimize the false negative rate (FNR). In NSABP - B32 trial clearly reported that removing 2 sentinel nodes rather than one, reduces the FNR by $50 \%$. Especially when used methylene blue dye alone it is important to follow the blue stain lymphatics and remove the suspicious nodes, closest nodes to the blue stained channels, at least 2 - 3 nodes that are qualified to be sentinel nodes rather than only the 'blue' and 'hot' node to reduce the FNR.

\section{Intraoperative evaluation of sentinel nodes}

Intraoperative evaluation of the sentinel node will allow immediate ALND if found positive, and to avert the need of a second surgery. It is understandable that many centres in Sri Lanka where SLND is practiced do not have resources for intraoperative evaluation. Further intraoperative evaluation of sentinel nodes adds time to already tight scheduled theatre hours in many Government / Public sector hospitals in Sri Lanka.

Although several intraoperative techniques can be used to identify a positive sentinel node such as cytology of node imprints [20], cytokeratin staining [21], and/or frozen sections [22], none of these methods will certainly identify all patients with positive nodes intraoperatively because of sampling limitations. While these limitations are considered, meticulous sampling techniques and an experienced pathologist can easily overcome the impact on FNR.

However, evaluation is more accurate on permanent (paraffin) sections which can minimize false negative rates and remains the gold standard. Therefore, the patients must be informed about the possibility of a second operation that may be needed for completion of nodal clearance, if intraoperative evaluation is not possible at their institutions.

\section{Pathology of sentinel nodes}

Sentinel node metastases are sub grouped into isolated tumor cell (ITC) clusters, micrometastases and macrometastases depending on the largest contiguous tumour deposit size, determined by routine hematoxylin and eosin stains. Immunohistochemistry staining is not routinely recommended to use in the assessment.

Isolated tumour cell clusters are defines as tumour cells less than $0.2 \mathrm{~mm}$ or nonconfluent or nearly confluent cluster of cells not exceeding 200 cells in a single lymph node cross section. ITC clusters are considered as N0 according to the Tumour, Node, and Metastasis (TNM) staging system. Micrometastases are defined when the deposits are more than $0.2 \mathrm{~mm}$ and less than $2 \mathrm{~mm}$ within the node and classified as Nmic according to TNM. As both N0 and Nmic do not have any prognostic significance, do not necessitate further surgical treatment to the axilla.

\section{Conclusion}

Sentinel lymph node dissection is the gold standard in the management of the axilla for early breast cancers. The recommended and globally accepted guidelines are provided by the National Comprehensive Cancer Network for all surgeons who treat breast cancers to follow evidence-based surgery. Sri Lanka been a country with limited resources, there are many meta-analyses to support techniques to conform to our best practice. As methylene blue dye alone is used at most institutions in Sri Lanka, it is recommended attempting to identify more sentinel nodes, removing any hard or large nodes found adjacent to them, only to perform routinely after the technique been validated by the surgeon and selecting patients with smaller tumors. Any technique not properly validated could hinder the cure rates of an early breast cancer.

All authors disclose no conflict of interest. The study was conducted in accordance with the ethical standards of the relevant institutional or national ethics committee and the Helsinki Declaration of 1975, as revised in 2000 .

\section{References}

1. Maunsell E, Brisson J, Deschenes L. Arm problems and psychological distress after surgery for breast cancer. Can J Surg 1993; 36:315-20.

2. Giuliano AE, Kirgan DM, Guenther JM, Morton DL. Lymphatic mapping and sentinel lymphadenectomy for breast cancer. Ann Surg 1994; 220: 391-398.

3. Krag DN, Anderson SJ, Julian TB, et al. Sentinel-lymph-node resection compared with conventional axillary-lymph-node dissection in clinically node-negative patients with breast 
cancer: overall survival findings from the NSABP B-32 randomised phase 3 trial. Lancet Oncol. 2010 Oct; 11(10):927-33. https://doi.org/10.1016/s1470-2045(10)70207-2

4. Lyman G H, Giuliano A E, Somerfield M R, et al. American Society of Clinical Oncology guideline recommendations for sentinel lymph node biopsy in early - stage breast cancer. J Clin Oncol 2005; 23:7703-7720.

https://doi.org/10.1200/jco.2005.08.001

5. Kuehn T, Vogl FD, Helms G, et al. Sentinel-node biopsy for axillary staging in breast cancer: results from a large prospective German multiinstitutional trial. Eur J Surg Oncol 2004; 30:252 259.

https://doi.org/10.1016/j.ejso.2003.11.016

6. Veronesi U, Paganelli G, Viale G, et al. A randomized comparison of sentinel-node biopsy with routine axillary dissection in breast cancer. N Engl J Med 2003; 349:546 - 553. https://doi.org/10.1056/nejmoa012782

7. Diepstraten S C, Sever A R, Buckens C F, et al. Value of preoperative ultrasound-guided axillary lymph node biopsy for preventing completion axillary lymph node dissection in breast cancer: a systematic review and meta-analysis. Ann Surg Oncol 2014 Jan;21(1):51-9.

https://doi.org/10.1245/s10434-013-3229-6

8. Mocellin S, Goldin E, Marchet A, Nitti D. Sentinel node biopsy performance after neoadjuvant chemotherapy in locally advanced breast cancer: A systematic review and meta-analysis. Int J Cancer. 2016;138(2):472.

https://doi.org/10.1002/ijc.29644

9. El Hage Chehade H, Headon H, El Tokhy O. Is sentinel lymph node biopsy a viable alternative to complete axillary dissection following neoadjuvant chemotherapy in women with nodepositive breast cancer at diagnosis? An updated meta-analysis involving 3,398 patients. Am J Surg. 2016 Nov;212(5):969-981. https://doi: 10.1016/j.amjsurg.2016.07.018

10. Klimberg VS, Rubio IT, Henry R, Cowan C, Colvert M, Korourian S. Subareolar versus peritumoral injection for location of the sentinel lymph node. Ann Surg. 1999;229(6):860. https://doi.org/10.1097/00000658-199906000-00013

11. Celebioglu F, Frisell J, Danielsson R, Bergkvist L. Sentinel node biopsy in non-palpable breast cancer and in patients with a previous diagnostic excision. Eur J Surg Oncol. 2007 Apr;33(3):276-80. Epub 2006 Dec 18. https://doi.org/10.1016/j.ejso.2006.11.008

12. Heuts EM, van der Ent FW, Kengen RA, van der Pol HA, HulsewéKW, Hoofwijk AG. Results of sentinel node biopsy not affected by previous excisional biopsy. Eur J Surg Oncol. 2007 Apr;33(3):276-80. https://doi.org/10.1016/j.ejso.2006.11.008
13. Intra M, TrifiròG, Viale G, Rotmensz N, Gentilini OD, Soteldo J, Galimberti V, Veronesi P, Luini A, Paganelli G, Veronesi U. Second biopsy of axillary sentinel lymph node for reappearing breast cancer after previous sentinel lymph node biopsy. Ann Surg Oncol. 2005;12(11):895.

https://doi.org/10.1245/aso.2005.10.018

14. Katherine E Posther, Linda A McCall, Peter W Blumencranz, et al. Sentinel Node Skills Verification and Surgeon Performance: Data From a Multicenter Clinical Trial for Early-Stage Breast Cancer. Ann Surg 2005 Oct; 242(4): 593-602. https://doi.org/10.1097/01.sla.0000184210.68646.77

15.Lyman GH, Giuliano AE, Somerfield MR, Benson AB 3rd, Bodurka DC, Burstein HJ, et al. American Society of Clinical Oncology guideline recommendations for sentinel lymph node biopsy in early-stage breast cancer. J Clin Oncol. 2005;23(30):7703-20. https://doi.org/10.1200/jco.2005.08.001

16.Kim T, Giuliano AE, Lyman GH. Lymphatic mapping and sentinel lymph node biopsy in early-stage breast carcinoma: a metaanalysis. Cancer. 2006;106(1):4.

https://doi.org/10.1002/cncr.21568

17.Jiyu Li, Xiao Chen, Ming Qi, Yanshuang Li. Sentinel lymph node biopsy mapped with methylene blue dye alone in patients with breast cancer: A systematic review and meta-analysis. PLoS One. 2018; 13(9): e0204364. https://doi.org/10.1371/journal.pone.0204364

18.Chris Tsopelas, Richard Sutton. Why Certain Dyes Are Useful for Localizing the Sentinel Lymph Nod J Nucl Med 2002; 43:1377-1382. PMID: 12368377

19.Harlow Seth P. Sentinel lymph node biopsy in breast cancer: Techniques.(Internet) Last updated Sep 19, 2019. Available from: https://www.uptodate.com/

20.Motomura K, Nagumo S, Komoike Y, Koyama H, Inaji H. Accuracy of imprint cytology for intraoperative diagnosis of sentinel node metastases in breast cancer. Ann Surg. 2008;247(5):839. https://doi.org/10.1097/sla.0b013e3181675855

21.Krishnamurthy S, Meric-Bernstam F, Lucci A, Hwang RF, Kuerer HM, Babiera G, Ames FC, Feig BW, Ross MI, Singletary E, Hunt KK, Bedrosian I. A prospective study comparing touch imprint cytology, frozen section analysis, and rapid cytokeratin immunostain for intraoperative evaluation of axillary sentinel lymph nodes in breast cancer. Cancer. 2009;115(7):1555. https://doi.org/10.1002/cncr.24182

22.Vanderveen KA, Ramsamooj R, Bold RJ. A prospective, blinded trial of touch prep analysis versus frozen section for intraoperative evaluation of sentinel lymph nodes in breast cancer. Ann Surg Oncol. 2008;15(7):2006.

https://doi.org/10.1245/s10434-008-9944-8 